\title{
Avaliação da Relação Entre a Climatologia, as Condições Sanitárias (Lixo) e a Ocorrência de Arboviroses (Dengue e Chikungunya) em Quixadá-CE no Período Entre 2016 e 2019
}

\author{
Nathiel de Sousa Silva ${ }^{1}$ (D), José Maria Brabo Alves $^{1}$ (D), Emerson Mariano da Silva ${ }^{1}$ (D), \\ Rafael Rocha Lima ${ }^{1}$ \\ ${ }^{1}$ Mestrado Profissional em Climatologia e Aplicações nos Países da CPLP e África, \\ Universidade Estadual do Ceará, Fortaleza, CE, Brasil.
}

Recebido em: 26 de Junho de 2020 - Aceito em: 4 de Julho de 2020

\begin{abstract}
Resumo
Este estudo considerou questões de epidemiologia, sanitarismo, clima e modelos matemáticos para resultados sobre casos de epidemias de Dengue e Chikungunya na cidade de Quixadá-Ce, Brasil, 2016-2019. Os dados epidemiológicos foram fornecidos pela Secretaria Municipal de Saúde. Os dados climáticos foram fornecidos pelo FUNCEME, INMET e INPE. As informações sobre condições sanitárias foram qualitativamente inferidas por entrevistas com os moradores do município, além de registros fotográficos e notícias compiladas em jornais / portais on-line. As informações epidemiológicas, climáticas e sociais foram utilizadas como banco de dados e regras de mecanismo do modelo estatístico nebuloso aplicado, que objetiva validação teórica, diagnóstico e predição de arboviroses, considerando essa multiplicidade de variáveis. As variáveis temperatura do ar e umidade relativa do ar permaneceram na zona de conforto do Aedes aegypti. A condição sanitária da cidade provou ser o principal fator de notificação dessas doenças durante o período do estudo, resultando em uma relação matemática diretamente proporcional entre casos confirmados de arboviroses e acúmulo de lixo. O sistema difuso aplicado foi assertivo próximo a $90 \%$, o que indica ser consistente e habilitado para os objetivos almejados, gerando diagnósticos e prognósticos para casos futuros dessas arboviroses, criando políticas públicas eficientes que atendam plenamente à população.
\end{abstract}

Palavras-chave: clima, arboviroses, lixo.

\section{Appraisal of the Relation Between Climatology, Sanitary Conditions (Garbage) and the Occurrence of Arboviroses (Dengue and Chikungunya) at Quixadá-Ce in the Period Between 2016 and 2019}

\begin{abstract}
This study was considerated questions from epidemiology, sanitarism, climate and mathematic models for results about epidemics cases of Dengue and Chikungunya at Quixadá City-Ce, Brazil, 2016-2019. The epidemiological data was provided by the Municipal Health Secretariat. Climate data was provided by FUNCEME, INMET and INPE. Information about sanitary condition was qualitatively infer with a series of interviews were conducted with residents of the municipality along with photographic records and compiled news in online newspapers/portals. The epidemiological, climatic and social information was used as a database and rules on engine of the fuzzy statistical model in application, which aims at theoretical validation, diagnosis and prediction of arbovirosis, considering this multiplicity of variables. The variables air temperature and relative air humidity remained within the comfort zone of Aedes aegypti. The city's sanitary condition's proved to be the main factor to notification of these diseases throughout the study period, resulting in a directly proportional mathematical relationship between confirmed cases of arbovirosis and garbage accumulation. The fuzzy system applied was assertive close to $90 \%$, which indicates that it is consistent and skilled for the objectives pursued generating diagnoses and prognoses for future cases of these arbovirosis, creating efficient public policies that fully meet population.
\end{abstract}

Keywords: climate, arbobirosis, garbage.

Autor de correspondência: Nathiel de Sousa Silva, nathiel.silva@uece.br. 


\section{Introdução}

A incidência de casos de Dengue e Chikungunya pode variar de acordo com as condições climáticas e do acúmulo de criadouros para o vetor (Aedes aegypti). Segundo a FUNASA (1999), esse mosquito possui grande importância epidemiológica, tendo difícil combate em todo território brasileiro. A diversidade de criadouros contribui diretamente na produção de indivíduos adultos, assumindo importante risco na dispersão de doenças (Rossi e Silva, 2007). Assim, é importante cruzar os diferentes dados para o direcionamento das ações de vigilância e tomada de decisões.

Depradine (2004) menciona o aumento no número desses insetos associado ao aumento da temperatura e da umidade relativa do ar, à pluviosidade e às condições sanitárias favoráveis ao mosquito. Para Ribeiro (2006), a expansão das áreas de ocorrência de Dengue no Brasil está associada à urbanização sem a devida estrutura de saneamento. Qualquer recipiente, material sólido, objeto, resíduo ou estrutura que possa acumular água, mesmo que em pequena quantidade, pode virar um criadouro.

A chuva é apontada como variável meteorológica de correlação positiva para essas doenças no Brasil segundo Viana e Ignotti (2013). Para Beserra et al. (2009), a temperatura do ar favorável ao desenvolvimento do mosquito encontra-se acima dos $22{ }^{\circ} \mathrm{C}$ e abaixo dos $32{ }^{\circ} \mathrm{C}$. Já Schweigmann et al. (2004), verificaram que as maiores proliferações dos mosquitos ocorreram durante meses com as maiores temperaturas do ar e precipitações registradas médias acima de $20^{\circ} \mathrm{C}$ e precipitações acumuladas acima de $150 \mathrm{~mm}$. Rodrigues (2004) indica que faixa favorável de umidade para os insetos é em torno de $40 \%$ a $80 \%$, o que indica uma maior velocidade de desenvolvimento, maior longevidade e maior fecundidade.

Em Quixadá-CE, existem diversos problemas, como nas condições sanitárias, porém tem se destacado como centro urbano cearense. Na região, o vírus da Dengue é prevalente há muitos anos e o vírus da Chikungunya gerou atenção desde o seu caso primário em 2016. O espalhamento e a persistência destas viroses estão condicionados à sobrevivência e reprodução do $A$. aegypti, no ambiente segundo Rizzi et al. (2017).

Conforme Varejãoet al.(2005), este vetor prefere reproduzir em reservatórios de águas limpas, embora possa se adaptar às novas situações impostas pelo homem, como por exemplo esgotos a céu aberto. Paralelamente, às ocorrências da doença e às dinâmicas climáticas e sociais, percebe-se a relevante atenção multidisciplinar de diversos pesquisadores com o tema em seus estudos científicoepidemiológicos com foco nessas avaliações.

Segundo Barsante (2012), pesquisas que utilizam conceitos de diversas áreas para descrever, analisar, monitorar e controlar a dinâmica espacial do ciclo de vida desses vetores tem fomentado a pesquisa mundialmente. De acordo com Barros et al. (1999), como os fenômenos biológicos possuem uma carga grande de subjetividade, a qual não é incorporada nos modelos matemáticos tradicionais determinísticos que os descrevem, buscou-se o uso da Teoria dos Conjuntos Difusos que contemplam esses graus de incertezas.

A teoria dos conjuntos fuzzy é um método usado para caracterizar, quantificar incertezas e imprecisões em dados e relações funcionais (Zadeh, 1965). Para Silva et al. (2007), a aplicação da teoria dos conjuntos difusos em estudos meteorológicos vem crescendo nos últimos anos por todo o mundo.

No contexto desse estudo, objetivou-se avaliar as condições climáticas e sanitárias observadas no município de Quixadá-CE e suas relações com as ocorrências das arboviroses (Dengue e Chikungunya) ocorridas na região, no período de 2016 a 2019. Em adição, avalia-se a aplicação de um modelo que usa a lógica dos conjuntos difusos (lógica fuzzy) para prever os cenários de ocorrências dessas arboviroses.

\section{Materiais e Métodos}

O presente estudo foi realizado no município brasileiro de Quixadá-CE. A região apresenta clima tropical quente semiárido com período chuvoso estabelecido de janeiro a maio, concentrando de fevereiro a abril.No município, os motores do clima são os mesmos que caracterizam as condições meteorológicas da região semiárida do nordeste brasileiro (Alves et al., 2009).

Existem sete postos pluviométricos distribuídos e realizando análises de precipitação em Quixadá-CE. Para Bertoni e Tucci (2002), há vários métodos para cálculo da precipitação média. Para esse estudo, usou-se o método de Thiesen (1911).

Os bancos de dados epidemiológicos, quanto aos agravos Dengue e Chikungunya de Quixadá-CE (20122019), foram cedidos pela Secretaria Municipal de Saúde no dia 29 de maio de 2019. As informações climáticas da região foram obtidas através da página eletrônica da Fundação Cearense de Meteorologia (FUNCEME) e do Instituto Nacional de Meteorologia (INMET). Os valores pluviométricos observados devem ser comparados aos esperados de acordo com a Normal Climatológica para a região e período de interesse.

Durante o processo de desenvolvimento da pesquisa, realizou-se uma série de entrevistas com a população local. As perguntas atuaram como motores para uma inferência qualitativa da situação de sanidade urbana em Quixadá-CE, devido à ausência de dados quantitativos quanto ao lixo e sua coleta na região.

Foi aplicado um modelo baseado na lógica fuzzy, baseado em Silva et al. (2007). A técnica foi exitosa no estudo de diagnóstico e previsão sazonal de chuva sobre a região nordeste do Brasil. Esse método permitiu um resul- 
tado determinístico, agregando informações climáticas ao perfil qualitativo inferido da situação sanitária em Quixadá-CE, para quantificar e prever as ocorrências das arboviroses na região.

Assim, possibilitam-se diferentes prognósticos, pertinentes à logica fuzzy, em categorias referentes ao número de casos das doenças como, por exemplo: BI (baixa incidência), MI (moderada incidência), AI (alta incidência), AAI (altíssima incidência) e EI (extrema incidência).

Nesse estudo, as variáveis difusas de entrada se referem às condições climáticas (pluviosidade, temperatura do ar e umidade relativa do ar) e ao estado qualitativo inferido do lixo em Quixadá-CE nos primeiros cinco meses de cada ano do estudo, como exposto no Quadro 1. A saída corresponde aos casos confirmados das arboviroses (Dengue e Chikungunya) na região. As informações climáticas observadas em cada mês/ano, presentes no Quadro 1, serviram de base para a definição das funções de pertinência das variáveis difusas de entrada.

Essas funções são definidas pela análise do número de variáveis climáticas com efeito positivo ao ciclo de vida do $A$. aegypti, tratadas aqui como eventos, considerando pesos iguais entre as mesmas. Assim, cada variável climática poderá apresentar os seguintes valores: (+), se positiva ao desenvolvimento do Aedes/Doenças; (-), se negativa ao desenvolvimento do Aedes/Doenças; (0), se neutra ao desenvolvimento do Aedes/Doenças.

Para tal, utilizou-se o conceito clássico de relação entre agravos à saúde como a Chikungunya e Dengue com variáveis climáticas definido de Time Lag. Segundo Depradine (2004), permite-se investigar os fenômenos resultantes das interações com o ambiente num determinado espaço de tempo.

Conforme Viana e Ignotti (2013), apesar das variações meteorológicas se apresentarem como um importante preditor, sabe-se que na maioria das situações existe uma defasagem na associação entre as variáveis meteorológicas

Quadro 1 - Condição das variáveis climatológicas, de janeiro-março, janeiro-abril e janeiro-maio, de cada ano considerado no estudo, usadas como preditoras do número de mosquitos e assim casos de Dengue e Chikungunya. As siglas significam: $\mathrm{T}^{\circ}$ para temperatura média do ar; $\mathrm{Ur}$ para umidade relativa do ar.

\begin{tabular}{lcccccc}
\hline Clima/ & JAN- & JAN- & JAN- & JAN- & JAN- & JAN- \\
período & MAR & ABR & MA & MAR & ABR & MAI \\
& 2016 & 2016 & 2016 & 2016 & 2016 & 2016 \\
\hline Chuva & - & - & - & + & + & + \\
$\mathrm{T}^{\circ}$ & + & + & + & + & + & + \\
Ur & + & + & + & + & + & - \\
Clima/ & JAN- & JAN- & JAN- & JAN- & JAN- & JAN- \\
período & MAR & ABR & MAI & MAR & ABR & MAI \\
& 2016 & 2016 & 2016 & 2016 & 2016 & 2016 \\
Chuva & + & + & + & + & + & + \\
$\mathrm{T}^{\circ}$ & + & + & + & + & + & + \\
Ur & + & + & + & + & + & + \\
\hline
\end{tabular}

e a ocorrência dessas doenças. Para Ferreira (2018), as incidências de dengue começam a aumentar no mês de janeiro, com os picos de chuva, tendo esse aumento na pluviosidade refletido nas taxas de incidências nos meses posteriores. Assim, definiu-se três períodos a serem avaliados em cada ano: janeiro a março; janeiro a abril e janeiro a maio.

A análise dos eventos (+), (-) e (0) definem as condições de entrada como Desfavoráveis (D), Neutras $(\mathrm{N})$ ou Favoráveis $(\mathrm{F})$, assumidas através da base de conhecimentos e as regras do motor de interferência do sistema fuzzy.

A definição para a entrada difusa quanto ao cenário das condições do lixo em Quixadá-CE será considerada como: MF (lixo Muito Favorável ao desenvolvimento do mosquito A.aegypti), F (lixo Favorável A. aegypti), N (lixo em condições de normalidade - sem maiores implicações para o desenvolvimento do mosquito) e $\mathrm{D}$ (cenário desfavorável para a proliferação do mosquito).

A variável de saída (número de casos de arboviroses - Dengue e Chikungunya) é produto da definição das regras definidas na base de regras e no motor de inferência do sistema fuzzy, como no Quadro 2.

As siglas indicam as saídas quanto às incidências das doenças: BI (baixa incidência), MI (moderada incidência), AI (alta incidência); AAI (altíssima incidência) e EI (extrema incidência).

O Programa Nacional de Controle de Dengue define: baixa incidência até 100 casos por 100 mil habitantes (hab), média incidência de 101 a 299 casos por 100 mil hab e alta incidência de 300 casos ou mais por 100 mil habitantes. Neste estudo, como o município de QuixadáCE possui, aproximadamente, 80.000 habitantes, considerou-se duas classes a mais como critérios para classificar a incidência dessas arboviroses.

Assim, para a variável fuzzy de saída definiram-se os seguintes cenários, como no Quadro 3.

Ressalta-se que os valores considerados para comparação nos resultados atendem à definição dos cenários acima, porém a saída desfuzzificada foi dada relativa aos limites das categorias da função triangular mostrada na Fig. 1. Em adição, no motor de inferência do sistema fuzzy assumiu-se, também, o conceito mínimo-máximo, considerado por Silva et al. 2007 que foi baseado em Zadeh (1965), os quais associam a função de pertinência $(\mu)$ ao somatório dos valores de cada variável difusa de entrada, assumindo-se a pertinência máxima para os cenários assumidos do clima e do lixo em Quixadá-CE.

Quadro 2 - Regras de inferência associadas às entradas observadas consoantes ao clima e coleta de lixo.

\begin{tabular}{lcccc}
\hline Clima/lixo & D & N & F & MF \\
\hline D & BI & BI & MI & EI \\
N & BI & MI & AI & EI \\
F & BI & MI & AAI & EI \\
\hline
\end{tabular}


Quadro 3 - Cenários de saída definidos para a variável fuzzy.

\begin{tabular}{ll}
\hline Número de casos & Categoria \\
\hline $0-50$ & BI (baixa incidência) \\
$50-100$ & MI (média incidência) \\
$100-200$ & AI (alta incidência) \\
$200-300$ & AAI (altíssima incidência) \\
acima de 300 & EI (extrema incidência) \\
\hline
\end{tabular}

No escopo desses cenários, considera-se, para cada, um conjunto de informações pertinentes, considerando os valores climáticos e sanitários: (BI), que sugere que as condições climáticas estão de minimamente favoráveis à favoráveis ao mosquito aliada a uma situação sanitária adequada, o que resulta em menos casos confirmados; (MI), que sugere que as condições climáticas podem estar minimamente favoráveis ao A.aegypti atiladas à condições sanitárias inadequadas; ademais, condições climáticas mais favoráveis ao mosquito com o lixo em situação moderada, as quais resultariam em mais casos sem entrar em categorias superiores alarmantes; (AI), que sugere que as condições climáticas estão adequadas ou minimamente adequadas ao A.aegypti com as condições sanitárias em estado inadequado; (AAI), se as condições sanitárias atingirem condições mais agravantes, os quais resultam em números expressivos de casos, tendo o lixo como um propulsor; (EI), que sugere situações em que o lixo encontrase em condições de alta gravidade aliado aos diferentes tipos de condições climáticas (mínimas à maximamente favoráveis ao A.aegypti), o que resultam em números extremos de confirmações de casos das doenças.

Para o somatório das arboviroses, considerando-se todo o período de dados da investigação (totais mensais de janeiro a maio para cada ano, de 2016 a 2019) foi registrado valor mínimo zero, médio de 229 e máximo de 602 casos. O período selecionado (janeiro a maio) compreende os meses da pré-estação e estação chuvosa na região. Os valores observados serviram de base para a definição da função de pertinência triangular da variável de saída fuzzy, mostrada na Fig. 1.

Para o período de janeiro a maio de 2016 (jan-mai/ 2016) observou-se o cenário desfavorável (com nível de pertinência 1) para a entrada climática, com precipitações acumuladas de 386 milímetros ( $\mathrm{mm}$ ) no período, que corresponde a $37 \%$ abaixo da média (normal) climatológica, porém com água suficientemente acumulada para o desenvolvimento do mosquito A.aegypti na região. Isto somado a definição do cenário do lixo como Muito Favorável (MF) a proliferação do mosquito, também com nível de pertinência 1 , retornou um cenário de saída fuzzy definido na Base de Regras como de EI (extrema incidência), como exposto no Quadro 4.

De acordo com a Base de Conhecimentos e considerando as regras definidas no Motor de Interferência, obtiveram-se os cenários definidos como variável de saída

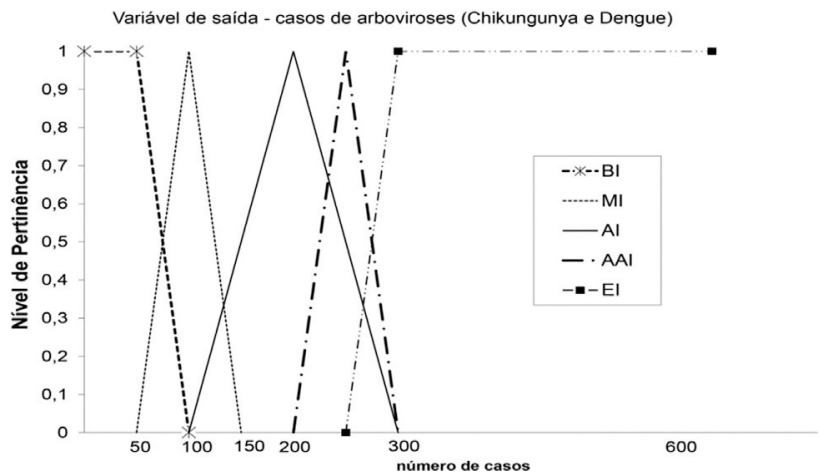

Figura 1 - Variável de saída quanto ao número de casos confirmados das arboviroses (Dengue e Chikungunya). Fonte: Elaborado pelo autor, adaptado de Silva et al. (2007).

Quadro 4 - Representação da situação de saída - Jan-maio/2016.

\begin{tabular}{lccccc}
\hline Clima/Lixo & $\mu$ & D & N & F & MF \\
\hline$\mu$ & EI, $\mu=1$ & & & & 1 \\
D & & 1 & & & \\
$\mathrm{~N}$ & & & & & \\
$\mathrm{~F}$ & & & & & \\
\hline
\end{tabular}

para cada período investigado, como mostrado no Quadro 4 para o exemplo do período de janeiro a maio de 2016. Para a desfuzzificação da variável de saída foi usado o método das máximas alturas médias, no qual calcula-se o valor médio entre os valores de máxima pertinência encontrados na variável difusa de saída.

Como exemplo, no período Janeiro-março/2018 foram observados 70 casos, o que se encaixa na classe MI. Dessa forma, o valor final ( $\mathrm{z}^{*}$ ) da saída desfuzzificada, usando o método das máximas alturas médias, foi dado pelos limites (a e b), mínimos e máximos, da função triangular dividido por dois. Assim, compreende-se a $=50 \mathrm{e}$ $\mathrm{b}=150$, sendo, então $\mathrm{z}^{*}=100$.

\section{Resultados e Discussões}

De forma geral, conforme a Fig. 2, foi constatado que as variáveis climáticas (temperaturas médias e máximas do ar e umidade relativa do ar média) não sofreram oscilações consideráveis durante toda a série temporal, contudo mantendo-se favoráveis ao A.aegypti. Assim, indica potencialidade a uma maior densidade populacional do mosquito, o que implica em maiores números de casos confirmados.

Já a pluviosidade aumentou rumo ao esperado na Normal Climatológica a partir do ano de 2017, acompanhada da redução no número de casos dessas doenças em comparação a 2016. Salienta-se que os anos com menores índices pluviométricos verificados (2016 e 2019) obtiveram maiores números de casos das doenças, tendo como 


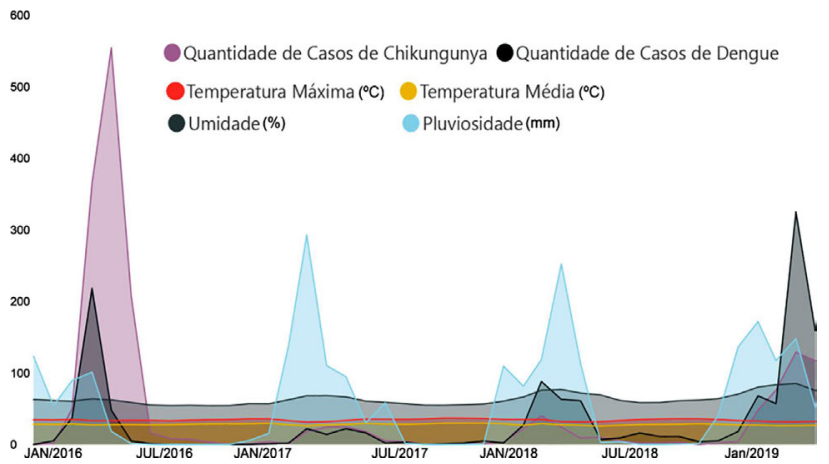

Figura 2 - Gráfico apresentando os comportamento das temperatura do ar máxima $\left({ }^{\circ} \mathrm{C}\right)$, temperatura do ar media $\left({ }^{\circ} \mathrm{C}\right)$, umidade relativa do ar média $(\%)$, pluviosidade $(\mathrm{mm})$ e dos números de casos confirmados de Dengue e Chikungunya em Quixadá de 2016 a maio de 2019.

justificativa, a situação sanitária da cidade em estado mais precário.

O clima isolado não rege a epidemiologia dessas doenças. Questões sociais, educativas e de gestão pública influenciam nesses processos, como a coleta regular e descarte adequado do lixo. Segundo Ebi (2016), a eliminação de criadouros propícios à reprodução de seu vetor é uma das mais importantes formas de prevenção.

Nos anos de 2017 e 2018, a situação sanitária precária foi mitigada e tornou a progredir na segunda parte do biênio, o que ainda resultou em um menor número de casos dos agravos à saúde mesmo apresentando maiores índices pluviométricos.

Em 2016, foram confirmados 1207 casos de Chikungunya em Quixadá-CE, havendo três óbitos. Sugere-se que essa altíssima incidência deveu-se a uma correlação positiva entre as condições climáticas mínimas para o vetor ligadas à máxima condição de acúmulo e ingerência dos resíduos sólidos, além da imunocompetência populacional.

Ressalta-se que 2016 foi o primeiro ano de notificação de Chikungunya em Quixadá-CE, não existindo então parâmetros anteriores para efeitos comparativos. No referido período (2016), a incidência da Chikungunya ultrapassou os 1000 casos por 100.000 habitantes, sendo superior à incidência da prevalente Dengue. Ressalta-se que a incidência representa a frequência com que surgem novos casos de uma determinada doença em um intervalo de tempo, considerando o total de pessoas expostas ao risco.

Em 2016, com maior número de casos para ambos agravos, a pluviosidade foi a menor observada em todo o período. Contudo, nos anos seguintes, a pluviosidade apresentou um aumento substancial acompanhada da redução expressiva no número de casos confirmados dessas enfermidades. O Quadro 5 mostra esses números.

Embasados nas análises das respostas da populaçãoalvo aos questionários, pode-se afirmar que houve o surgi- mento de maiores cuidados profiláticos pela população da região sobre as doenças e a adoção de corretos procedimentos de estocagem de águas nos domicílios; absorção das iniciativas sócioeducativas pelas entidades governamentais municipais; evidenciação das seqüelas e óbitos provenientes da doença como forma de alerta para os habitantes da área de estudo.

As respostas aos questionários atestaram à insatisfação popular com a limpeza pública em 2016 e uma satisfação no biênio 2017/2018, mesmo com o retorno, ainda em 2018, às condições sanitárias preocupantes. O desconforto populacional voltou a ser atestado entre 2018-2019 com o retorno do lixão e retomada da precariedade na gestão de resíduos sólidos do município.

Destaca-se que em meio ao período compreendido por esse estudo, em 2017, houve a extinção do lixão de Quixadá-CE, tendo sido o seu material destinado ao aterro sanitário do município de Senador Pompeu-CE, que distam aproximadamente 100 quilômetros, o qual consoante a Sales et al. (2019), é o primeiro aterro ecologicamente correto da região com $100 \%$ de investimentos da iniciativa privada. Essa ação apresenta-se relevante na redução das moléstias na região, visto que esses resíduos serviam de potenciais berçários para o mosquito transmissor. O lixão em progressão não indica ser o único local criadouro do mosquito, mas serve de informação para o diagnóstico da precariedade sanitária da cidade, a qual, sim, demonstra correlação positiva e direta com o aumento na incidência de agravos à saúde.

Borja e Moraes (2003) destacam a importância dos indicadores em planejamento de saúde como instrumentos capazes de auxiliar os gestores a avaliar os resultados do trabalho desenvolvido e redefinir o melhor rumo das políticas públicas. Dessa forma, de acordo com Sousa-Santos (1999), surge a necessidade de maior atenção sobre a importância do correto acondicionamento dos resíduos Porém, para Lefèvre (2007), tão importante quanto acondicionar corretamente o lixo, está a coleta eficiente e regular, uma vez que se relaciona diretamente com o controle do vetor.

Quadro 5 - Relação dos casos de Chikungunya e Dengue confirmadas e pluviosidade média em Quixadá-CE no período 2016-2019.

\begin{tabular}{ccccc}
\hline Ano & Chikungunya & Dengue & $\begin{array}{c}{ }^{*} \text { Pluviosidade } \\
\text { anual }(\mathrm{Mm})\end{array}$ & $\begin{array}{c}{ }^{*} \text { Pluviosidade Jan/ } \\
\text { Maio (Mm) }\end{array}$ \\
\hline & & & & \\
2016 & 1.207 & 314 & 392,9 & 386,1 \\
2017 & 99 & 86 & 743,4 & 652,5 \\
2018 & 110 & 293 & 723,8 & 675 \\
2019 & ${ }^{* *} 289$ & ${ }^{* *} 630$ & - & 625 \\
\hline
\end{tabular}

Fontes: FUNCEME e Secretaria Municipal de Saúde.

*Pluviosidade média considerando o método Thiessen.

**Apresentam alguns casos ainda em investigação na data do envio dos dados epidemiológicos. 
Em 2019, as arboviroses tiveram novo aumento em número de casos novos e os índices pluviométricos mantiveram-se similares aos do biênio anterior. Contudo, ressurgiu, de forma acentuada, a problemática do lixo urbano. De forma geral, a situação inferida para a questão do acúmulo de lixo em Quixadá é apresentada a seguir:

a. Em 2016, a cidade encontrava-se em situação Favorável ao desenvolvimento do $A$. aegypti e suas arboviroses;

b. Em 2017, a cidade encontrava-se em situação Desfavorável ao desenvolvimento do $A$. aegypti e suas arboviroses;

c. Em 2018, a cidade encontrou-se em situação transitória de Desfavorável a Favorável ao desenvolvimento do $A$. aegypti e suas arboviroses;

d. Em 2019, a cidade encontrava-se em situação Favorável ao desenvolvimento do $A$. aegypti e suas arboviroses.

Condições climáticas e sanitárias (acumulo de lixo, limpeza pública e existência de microlixões) consideradas para a validação do método fuzzy proposto por Silva et al. (2007) são apresentadas, como exemplos, no Quadro 6.

Definiu-se a combinação das variáveis de entrada com a saída fuzzy, a exemplo no caso de Jan-Mar/2016 Clima Desfavorável (D) e Lixo Muito Favorável (MF) para uma saída de Extrema Incidência (EI) no número de caso das arboviroses.

O resultado após a aplicação da regra de inferência, de acordo com os outros resultados analíticos das variáveis de entrada, com as suas respectivas categorias e níveis de pertinência $(\mu)$ para os anos períodos de janeiro-março, janeiro-abril e janeiro-maio de cada ano do período 20162019 estão representado no Quadro 7.

De acordo com Silva et al. (2007), o método no qual esse estudo é baseado visa otimizar as informações advindas das interpretações e diferentemente da aplicação de métodos estatístico-estocástico de previsão não é necessário um período de treinamento e outro de validação do modelo.

Por fim, apresenta-se uma comparação entre os valores de saída (casos das doenças) observados e os valores otimizados encontrados usando conjuntos difusos por categorias, comparando-as entre si, como exposto no Quadro 8.

A exemplo, no período de janeiro a maio de 2017 (jan-mai/2017) observou-se o cenário Favorável (com nível de pertinência 1) para a entrada climática, com precipitações acumuladas de 652,5 milímetros $(\mathrm{mm})$ no período, que corresponde a $7 \%$ acima da média (Normal)

Quadro 6 - Exemplos de relações entrada/saída no sistema proposto.

\begin{tabular}{lccc}
\hline Condições/período & Jan-Março/2016 & Jan-Abril/2017 & Jan-Maio/2019 \\
\hline Climáticas & D & F & F \\
Lixo & MF & D & F \\
Saída Fuzzy & EI & BI & AI \\
\hline
\end{tabular}

Quadro 7 - Resultados da regra de inferência de acordo com os resultados do somatório das variáveis de entrada - saídas para decodificação.

\begin{tabular}{|c|c|c|c|c|c|c|c|c|c|c|c|}
\hline \multicolumn{6}{|c|}{ Jan-Mar/2016 } & \multicolumn{6}{|l|}{ Jan-Mar/2017 } \\
\hline Clima/Lixo & $\mu$ & D & $\mathrm{N}$ & $\mathrm{F}$ & MF & Clima/Lixo & $\mu$ & $\mathrm{D}$ & $\mathrm{N}$ & $\mathrm{F}$ & MF \\
\hline$\mu$ & EI & & & & 1 & $\mu$ & BI & 1 & & & \\
\hline $\mathrm{D}$ & & 1 & & & & $\mathrm{D}$ & & & & & \\
\hline $\mathrm{N}$ & & & & & & $\mathrm{N}$ & & & & & \\
\hline $\mathrm{F}$ & & & & & & F & & 1 & & & \\
\hline Jan-Abr/2016 & & & & & & Jan-Abr/2017 & & & & & \\
\hline Clima/Lixo & $\mu$ & $\mathrm{D}$ & $\mathrm{N}$ & $\mathrm{F}$ & MF & Clima/Lixo & $\mu$ & $\mathrm{D}$ & $\mathrm{N}$ & $\mathrm{F}$ & MF \\
\hline$\mu$ & EI & & & & 1 & $\mu$ & BI & 1 & & & \\
\hline $\mathrm{D}$ & & 1 & & & & $\mathrm{D}$ & & & & & \\
\hline $\mathrm{N}$ & & & & & & $\mathrm{N}$ & & & & & \\
\hline $\mathrm{F}$ & & & & & & $\mathrm{F}$ & & 1 & & & \\
\hline Jan-Mai/2016 & & & & & & Jan-Mai/2017 & & & & & \\
\hline Clima/Lixo & $\mu$ & $\mathrm{D}$ & $\mathrm{N}$ & $\mathrm{F}$ & MF & Clima/Lixo & $\mu$ & $\mathrm{D}$ & $\mathrm{N}$ & $\mathrm{F}$ & MF \\
\hline$\mu$ & EI & & & & 1 & $\mu$ & BI & 1 & & & \\
\hline $\mathrm{D}$ & & 1 & & & & $\mathrm{D}$ & & & & & \\
\hline $\mathrm{N}$ & & & & & & $\mathrm{N}$ & & & & & \\
\hline $\mathrm{F}$ & & & & & & $\mathrm{F}$ & & 1 & & & \\
\hline Jan-Mar/2018 & & & & & & Jan-Mar/201C & & & & & \\
\hline Clima/Lixo & $\mu$ & $\mathrm{D}$ & $\mathrm{N}$ & $\mathrm{F}$ & MF & Clima/Lixo & $\mu$ & $\mathrm{D}$ & $\mathrm{N}$ & $\mathrm{F}$ & MF \\
\hline$\mu$ & AI & & 1 & & & $\mu$ & AAI & & & 1 & \\
\hline $\mathrm{D}$ & & & & & & $\mathrm{D}$ & & & & & \\
\hline $\mathrm{N}$ & & & & & & $\mathrm{N}$ & & & & & \\
\hline $\mathrm{F}$ & 1 & & & & & $\mathrm{~F}$ & 1 & & & & \\
\hline Jan-Abr/2018 & & & & & & Jan-Abr/2019 & & & & & \\
\hline Clima/Lixo & $\mu$ & $\mathrm{D}$ & $\mathrm{N}$ & $\mathrm{F}$ & MF & Clima/Lixo & $\mu$ & $\mathrm{D}$ & $\mathrm{N}$ & $\mathrm{F}$ & MF \\
\hline$\mu$ & MI & & & & & $\mu$ & AAI & & & 1 & \\
\hline $\mathrm{D}$ & & & & & & $\mathrm{D}$ & & & & & \\
\hline $\mathrm{N}$ & & & 1 & & & $\mathrm{~N}$ & & & & & \\
\hline $\mathrm{F}$ & 1 & & & & & $\mathrm{~F}$ & 1 & & & & \\
\hline Jan-Mai/2018 & & & & & & Jan-Mai/2019 & & & & & \\
\hline Clima/Lixo & $\mu$ & D & $\mathrm{N}$ & $\mathrm{F}$ & MF & Clima/Lixo & $\mu$ & $\mathrm{D}$ & $\mathrm{N}$ & $\mathrm{F}$ & MF \\
\hline$\mu$ & MI & & & & & $\mu$ & AAI & & & 1 & \\
\hline $\mathrm{D}$ & & & & & & $\mathrm{D}$ & & & & & \\
\hline $\mathrm{N}$ & & & 1 & & & $\mathrm{~N}$ & & & & & \\
\hline $\mathrm{F}$ & 1 & & & & & $\mathrm{~F}$ & 1 & & & & \\
\hline
\end{tabular}

climatológica. Isto somado a definição do cenário do lixo como Desfavorável (D) a proliferação do mosquito, também com nível de pertinência 1 , retornou um cenário de saída fuzzy definido na Base de Regras como de BI (baixa incidência), tendo a questão do lixo como o responsável pelo menor número de casos mesmo com o clima favorável.

Observa-se que a relação entre as entradas é consistente na obtenção de um retrato do estudo de caso, apontando somente dois casos particulares que possuem suas 
Quadro 8 - Período, número de casos, categoria observada, categoria do diagnóstico, valor máximo, valor obtido no método de desfuzzificação para os critérios subjetivos, desvio \% em relação ao número de casos desfuzzificados para os critérios subjetivos; valor obtido no método de desfuzzificação para critérios referidos na função triangular, desvio \% em relação ao número de casos desfuzzificados para critérios referidos na função triangular.

\begin{tabular}{|c|c|c|c|c|c|c|c|c|}
\hline Período & $\mathrm{N}^{\mathrm{o}}$ de casos & Cat. obs. & Cat. diag. & Val. máx. & Val. mét. subj. & Des\% fuz. sub. & Val. met. tri. & Des $\%$ fuz. tri. \\
\hline Jan-Mar/16 & 93 & MI* & EI & 600 & 75 & 24 & 100 & -7 \\
\hline JAN-ABR/16 & 583 & EI & EI & 600 & 450 & 29,5 & 425 & 37,17647 \\
\hline JAN-MAIO/16 & 602 & EI & EI & 600 & 450 & 33,7 & 425 & 41,64706 \\
\hline Jan-Mar/17 & 48 & BI & $\mathrm{BI}$ & 50 & 50 & -4 & 50 & -4 \\
\hline JAN-ABR/17 & 38 & BI & $\mathrm{BI}$ & 50 & 50 & -24 & 50 & -24 \\
\hline JAN-MAIO/17 & 47 & $\mathrm{BI}$ & $\mathrm{BI}$ & 50 & 50 & -6 & 50 & -6 \\
\hline Jan-Mar/18 & 182 & AI & AI & 200 & 150 & 21,3 & 200 & -9 \\
\hline JAN-ABR/18 & 87 & MI & MI & 100 & 75 & 16 & 100 & -13 \\
\hline JAN-MAIO/18 & 70 & MI & MI & 100 & 75 & $-6,66$ & 100 & -30 \\
\hline Jan-Mar/19 & 271 & AAI & AAI & 300 & 250 & 8,4 & 250 & 8,4 \\
\hline JAN-ABR/19 & 454 & EI* & AAI & 300 & 450 & 0,88 & 425 & 6,823529 \\
\hline JAN-MAIO/19 & 276 & AAI & AAI & 300 & 250 & 10,4 & 250 & 10,4 \\
\hline
\end{tabular}

devidas hipóteses de fuga ao padrão de êxito observado nos demais:

a. Janeiro-Março/2016. Esperava-se o enquadramento na categoria EI (extrema incidência). Contudo, acredita-se que a anomalia apresentada quanto ao grande número de casos de Chikungunya, em seu primeiro ano, tenham sub notificados e diagnósticos inconclusivos ou confundidos com outros agravos já prevalentes na região;

b. Janeiro-Abril/2019. Esperava-se enquadramento na categoria AAI (altíssima incidência), visto as entradas estarem ambas Favoráveis e analisadas de acordo com o motor de inferência proposto. Todavia, os números mais expressivos, subindo-a em categoria, possivelmente, devem-se ao tratamento dos dados ainda em curso pela Secretaria Municipal de Saúde de Quixadá e aos casos em investigação.

Os resultados mostrados no Quadro 7 indicam que, em 2016 por exemplo, o lixo foi decisivo para a apresentação do perfil epidemiológico observado, visto que o clima está normalmente favorável ao A. aegypti. E essa característica é consistente com todos os períodos estudados e referenciada de acordo com os trabalhos de Sobral e Sobral (2019), França et al. (2017) e Azeredo (2007).

\section{Considerações Finais}

As condições climáticas (pluviosidade, temperatura do ar e umidade relativa do ar) no período em estudo se apresentaram como favoráveis à proliferação do $A$. aegypti $\mathrm{e}$, consequentemente, às ocorrências de arboviroses em Quixadá/CE.

As condições sanitárias, referentes ao acúmulo de lixo como potencialdepósito de água contribuinte para a proliferação do vetor, mostraram-se variáveis cruciais no processo de modelagem e quantificação das arbovirores.
A avaliação de um modelo baseado em lógica fuzzy, com as variáveis propostas nesse estudo,foimuito satisfatória para inferir as classes e aproximar os quantitativos de casos das ocorrências de arboviroses na região.

É fato que a redução considerável nas confirmações de casos Dengue e Chikungunya em Quixadá, em 20172018, indicam que as transformações sociais e culturais foram fatores robustos para essa realidade epidemiológica, desmitificando a pluviosidade como causa única para o agravamento na incidência dessas mazelas.

Dessa forma, suscita-se a sinergia de diversos campos metodológicos e contextos, visando aumentar a compreensão dos fatores e eventos que possam interferir na saúde dos seres humanos. Aventam-se então, em futuros estudos, constantes análises e uma cooperação multidisciplinar para esses traçados epidemiológicos, respeitando o dinamismo com que a natureza se comporta. Outrossim, deve-se sempre considerar o comportamento da sociedade, o qual influencia direta e indiretamente nesses processos.

\section{Referências}

ALVES, J.M.B.; SERVAIN, J.; CAMPOS, J.N.B. Relationship Between Ocean Climate Variability and Rain Fed Agriculture in Northeast Brazil. Clim Res, v. 38, n. 3, p. 225236, 2009.

AZEREDO, C.M.; COTTA, R.M.M.; SCHOTT, M.; MAIA, T.M; MARQUES, E.S. Avaliação das condições de habitação e saneamento: a importância da visita domiciliar no contexto do Programa da Saúde da Família. Ciência Saúde Coletiva, v. 12, n. 7, p. 746-751, 2007.

BARROS, L.C.; BASSANEZI, R.C.;LEITE, M.B.F. Epidemiologia e Teoria Fuzzy. Biomatemática, v. IX, p. 40, 1999.

BARSANT, L.S. Dependência entre Pluviosidade e População de Fêmeas Aedes aegypti Grávidas Descritas Através de um Sistema Dinâmico Não Linear. Dissertação de Mes- 
trado em Modelagem Matemática e Computacional, Centro Federal de Educação Tecnológica de Minas Gerais, Belo Horizonte, p. 123, 2012.

BERTONI, J.C.; TUCCI, C.E.M. Precipitação. Hidrologia: Ciência e Aplicação. Organizado por TUCCI, C.E.M., Porto Alegre: Ed. Universidade, v. 4, p. 943, 2002.

BESERRA, E.B.; FREITAS, E.M,.; SOUSA, J.T.; FERNANDES, C.R.M.; SANTOS, K.D. Ciclo de vida de Aedes (Stegomyia) aegypti (Diptera, Culicidae) em águas com diferentes características. LheringiaSérie Zooloogia., v. 99, n. 3, p. 281-285, 2009.

BORJA, P.C.; MORAES, L.R.S. Indicadores de saúde ambiental com enfoque para a área de saneamento. Parte 1 - aspectos conceituais e metodológicos. Revista Engenharia Sanitária e Ambiental, v. 8, n.1, p. 13-25, 2003.

DEPRADINE C.; LOVELL, E. Climatological variables and the incidence of dengue fever in Barbados. Int $\mathbf{J}$ Environ Health Res., v. 14, n.6, p. 429-441, 2004.

DONALÍSIO, M.R.; GLASSER, C.M. Vigilância entomológica e controle de vetores do dengue. Rev Bras Epidemiol., v. 5, n. 3, p. 259-279, 2002.

EBI, K.L.; NEALON, J. Dengue in a changing climate. Environ Res, v. 151, p. 115-123, 2016.

FERREIRA, A.C.; CHIARAVALLOTI NETO, F.; MONDINI, A. Dengue em Araraquara, SP: epidemiologia, clima e infestação por Aedes aegypti. Revista de Saúde Pública, v. 52, n. 18, p. 1-10, 2018.

FRANÇA, L.S. et al. desafios para o controle e prevenção do mosquito Aedes aegypti. Rev Enferm, UFPE on-line., v. 11, n. 12, p. 4913-8, 2017.

FUNCEME. Fundação Cearense de Meteorologia. Disponível em www.funceme.br/,acesso em 10 de fev. de 2020.

FUNASA (FUNDAÇÃO NACIONAL DE SAÚDE), Manual de Vigilância Epidemiológica de Febre Amarela. Brasília: Funasa, p. $9,1999$.

GIL, L.H.S.; KATSURAGAWA, T.H.; LIMA, A.A.; TADA, M.S.; OZAKI, L.S.; JULIÃO, G.R. Rudimentary cesspits as breeding sites for Aedes aegypti in urban areas of Northern Brazil. Rev Pan-Amaz Saúde, v. 6, n. 3, p. 73-80, 2015.

GLASSER, C.M.; GOMES, A.C. Infestação do Estado de São Paulo por Aedes aegypti e por Aedes albopictus. Rev Saúde Pública, v. 34, n. 6, p. 570-577, 2001.

GOMES, A.C. Vigilância entomológica. Inf. Epidemiol. Sus, v. 11, n. 2, p. 79-90, 2002.

INPE. Instituto Nacional de Pesquisas Espaciais. Disponível em www.inpe.br/, acesso em 10 de fev. de 2020.

LEFÈVRE A.M.C.; RIBEIRO, A.F; MARQUES, G.R.A.M.; SERPA, L.L.N.; LEFÈVRE, F. Representações sobre dengue, seu vetor e ações de controle por moradores do município de São Sebastião, litoral Norte do Estado de São Paulo, Brasil. Cad. Saúde Pública, v. 23, n. 7, p. 16961706, 2007.

MARINHO, R.A. Ecobiologia de Aedes aegypti (L.1762) (Diptera: Culicidae) Associada a Fatores Climáticos em Três Mesorregiões da Paraíba. Dissertação de Mestrado em Ecologia e Conservação, Universidade Estadual da Paraíba, Campina Grande, p. 145, 2013.
MEDEIROS, L.C.C. et al. Modeling the dynamic transmission of dengue fever: investigating diseases persistence. Plos Neglected Tropical Disease, v. 5, n. 1, e942, p. 1-14, 2011.

PEREIRA, A.Q. Caracterização das atividades terciárias no centro urbano de Quixadá. BGG, v.41, n.1, p. 168-184, 2014.

RIBEIRO, A.F.; MARQUES, G.R.A.M.; VOLTOINI, J.C.; CONDINO, M.L.F. Associação entre incidência de dengue e variáveis climáticas. Rev Saúde Pública, v. 40, n. 4, p. 671-676, 2006.

RIZZI, C.B.; RIZZI, R.L.; PRAMIU, P.V.; HOFFMANN, E.; CODEÇO, C.T. Considerações sobre a dengue e variáveis de importância à infestação por Aedes aegypti. Hygeia, v. 13, n. 24, p. 24-40, 2017.

RODRIGUES, W.C. Fatores que influenciam no desenvolvimento dos insetos. Info Insetos, v.1, n.4, p. 1-4, 2004.

ROSSI, J.C.N.; SILVA, A.M. Diversidade de Criadouros Frequentados por Aedes aegypti e Aedes albopictus no Estado de Santa Catarina, Período de 1998 a 2007, Santa Catarina: Secretaria de Saúde,p.3, 2007.

SALES, C.H.N.; MARTINS, I.C.; ALMEIDA, A.M.; MATTOS, S.M. Diagnóstico da degradação ambiental na área do lixão de um Município no Sertão Central do Ceará. Encontro de Extensão, Docência e Iniciação Científica, Unicatólica,p. 3, 2019.

SCHWEIGMANN, N; VEZZANI, D.; VELÁZQUEZ, S.M. Seasonal Pattern of Abundance of Aedes aegypti (Diptera: Culicidae) in Buenos Aires City, Argentina. Revista do Instituto Oswaldo Cruz, v. 99, n. 4, p.351-356, 2004.

SILVA, E.M.; ALVES, J.M.B.; CASTRO, M.A.H.; VIEIRA, P.P.B.; CAMPOS, J.N.B. Uma aplicação de conjuntos difusos na otimização do prognóstico de consenso sazonal de chuva no Nordeste do Brasil. Rev. Bras. Meteorol., v. 22, n. 1, p. 83-93, 2007.

SOBRAL, M.F.F.; SOBRAL, A.I.G. Casos de dengue e coleta de lixo urbano: um estudo na Cidade do Recife. Ciências e Saúde Coletiva, v. 24, n. 3, p. 1075-1082, 2019.

SOUZA-SANTOS, R.Fatores associados à ocorrência de formas imaturas de Aedes aegypti na Ilha do Governador, Rio de Janeiro, Brasil.Rev. Soc. Bras. Med. Trop., v. 32, n. 4 p.373-382, 1999.

THIESSEN, A.H. Precipitation averages for large areas. Monthly Weather Review, v. 39, n.7, p. 1082-1089, 1911.

VAREJÃO, J.B.M.; SANTOS, C.B.; REZENDE, H.R.; BEVILACQUA, L.C.; FALQUETO, A. Criadouros de Aedes (Stegomyia) aegypti (Linnaeus, 1762) em bromélias nativas na cidade de Vitória, ES.Revista da Sociedade Brasileira de Medicina Tropical, v. 38, n. 3, p. 238-240, 2005.

VIANA, D.V.; IGNOTTI, E. A ocorrência da dengue e variações meteorológicas no Brasil: Revisão sistemática. Rev Bras Epidemiol., v. 16, n. 2, p. 240-256, 2013.

ZADEH, L.A. Fuzzy sets. Information and Control, v. 8, p. 338-353, 1965.

License information: This is an open-access article distributed under the terms of the Creative Commons Attribution License (type CC-BY), which permits unrestricted use, distribution and reproduction in any medium, provided the original article is properly cited. 